\title{
Childhood leukaemia and distance from power lines in California: a population-based case-control study
}

\author{
Catherine M Crespi ${ }^{*, 1}$, Ximena P Vergara ${ }^{2}$, Chris Hooper ${ }^{3}$, Sona Oksuzyan ${ }^{4}$, Sheng Wu ${ }^{1}$, Myles Cockburn ${ }^{5}$ \\ and Leeka Kheifets ${ }^{4}$ \\ ${ }^{1}$ Department of Biostatistics, University of California Los Angeles Fielding School of Public Health, 650 Charles E. Young Drive \\ South, Los Angeles, CA 90095-1772, USA; ${ }^{2}$ Environment Sector, Electric Power Research Institute, Palo Alto, CA 94304, USA; \\ ${ }^{3}$ Enertech Consultants, Campbell, CA 95008, USA; ${ }^{4}$ Department of Epidemiology, UCLA Fielding School of Public Health, \\ University of California Los Angeles, Los Angeles, CA 90095-1772, USA and ${ }^{5}$ Department of Preventive Medicine, University of \\ Southern California, Los Angeles, CA 90032, USA
}

Background: Studies have reported an increased risk of childhood leukaemia associated with living near high-voltage electric power transmission lines that extend to distances at which magnetic fields from lines are negligible. We conducted a large records-based case-control study of childhood leukaemia risk in the population living near power lines in California.

Methods: The study included 5788 childhood leukaemia and 3308 central nervous system (CNS) cancer cases (for comparison) born in and diagnosed in California (1986-2008), and matched to population-based controls by age and sex. We geocoded birth address and estimated the distance from residence to transmission lines using geographic information systems, aerial imagery, and, for some residences, site visits.

Results: For leukaemia, there was a slight excess of cases within $50 \mathrm{~m}$ of a transmission line over $200 \mathrm{kV}$ (odds ratio 1.4, 95\% confidence interval 0.7-2.7). There was no evidence of increased risk for distances beyond $50 \mathrm{~m}$, for lower-voltage lines, or for CNS cancers.

Conclusions: Our findings did not clearly support an increased childhood leukaemia risk associated with close proximity ( $<50 \mathrm{~m})$ to higher voltage lines, but could be consistent with a small increased risk. Reports of increased risk for distances beyond $50 \mathrm{~m}$ were not replicated.

The possibility that the electric power transmission and distribution system could pose a risk for childhood cancer has been a concern for several decades, beginning with the study of Wertheimer and Leeper (1979), which found an association with electrical wiring configurations. Since then, over 30 epidemiologic studies have investigated the association of childhood leukaemia with residential exposure to magnetic fields (Kheifets and Shimkhada, 2005). Pooled analyses combining the accumulating studies have reported an increased risk of childhood leukaemia associated with exposure to relatively high levels of magnetic fields based on in-home measurements and calculated fields generated by high-voltage power transmission lines near the residence (Ahlbom et al, 2000; Greenland et al, 2000; Kheifets et al, 2010).

Although magnetic field strength depends on distance from power lines, distance from lines has emerged as an exposure of interest in its own right. One of the key studies to investigate distance from power lines and childhood cancer risk was the study of Draper et al (2005). These authors reported a case-control study of childhood cancer in relation to the distance from birth address to the nearest high-voltage overhead transmission line (mostly of

*Correspondence: Dr CM Crespi; E-mail: ccrespi@ucla.edu

Received 19 February 2016; revised 6 April 2016; accepted 26 April 2016; published online 24 May 2016

(c) 2016 Cancer Research UK. All rights reserved 0007-0920/16 
275 or $400 \mathrm{kv}$ ) among children born in England and Wales in 1962-1995. They found an elevated odds ratio (OR) of 1.7 for childhood leukaemia among subjects very close to lines $(<50 \mathrm{~m})$ compared with those residing beyond $600 \mathrm{~m}$, which could be attributable to the magnetic fields exposure. However, they also found elevated risk extending out to $600 \mathrm{~m}$. Because magnetic fields generated by overhead lines drop rapidly with distance and are negligible beyond $200 \mathrm{~m}$ (Kaune and Zaffanella, 1992), the finding of increased risk at farther distances was difficult to explain.

A pooled analysis of six studies on the relation between childhood leukaemia risk and distance of the residence from power lines of various voltages was conducted by Kheifets et al (2010). They reported ORs of 1.6, 1.3, and 1.2 for distance to nearest line of $0-50,50-100$, and $100-200 \mathrm{~m}$, respectively, compared with $>200 \mathrm{~m}$, which leaves open the possibility of a small increased risk at distances beyond the influence of magnetic fields.

The Geocap study in France (Sermage-Faure et al, 2013), using methods similar to Draper et al (2005), observed increased ORs for childhood acute leukaemia for home address at diagnosis within $50 \mathrm{~m}$ of a high-voltage power line $(225-400 \mathrm{kV})$, and no association with living beyond $50 \mathrm{~m}$ from $225-400 \mathrm{kV}$ lines or within $50 \mathrm{~m}$ of 63-150 kV lines. Pedersen et al (2014), reporting a small study in Denmark, found no pattern of increased risk with proximity to lines. The statistical power of these studies was limited due to small numbers of subjects close to lines.

Bunch et al (2014) updated the study by Draper et al (2005), adding more recent cases and controls, extending the distance evaluation to $1000 \mathrm{~m}$ from lines, adding lower voltages, and examining temporal trends by decade. They found that the excess risk for leukaemia at distances out to $600 \mathrm{~m}$ declined from the 1960s through 2008, becoming null in more recent decades. However, the association in earlier decades remained unexplained.

We report here on the California Power Line Study (CAPS), the first large-scale study to focus on childhood leukaemia risk in the population living near transmission lines in the United States. CAPS is a population-based case-control study focusing on childhood leukaemia and including childhood central nervous system (CNS) cancer for comparison. We present analysis of childhood leukaemia and CNS cancer risk in relation to the distance from birth address to nearby power transmission lines. Results for calculated magnetic fields will be presented in a future report.

\section{MATERIALS AND METHODS}

Cases and controls. As previously described (Kheifets et al, 2015), the California Cancer Registry (CCR; www.ccrcal.org), a statewide population-based cancer registry, was used to obtain information on all childhood leukaemia and CNS cancer cases diagnosed between 1988 and 2008 among children younger than 16 years of age who were residing in California at the time of diagnosis. Beginning in January 1988, California law has required all new cancer cases diagnosed in state residents to be reported to the CCR. CCR data meet all standards of the Surveillance, Epidemiology and End Results Program and National Program of Cancer Registries for quality, timeliness, and completeness. Data collected by the registry include patient's name, address at time of diagnosis, sex, age at diagnosis, and type of cancer.

A total of 6645 childhood leukaemia and 3858 childhood CNS cancer cases meeting the inclusion criteria were identified from the CCR. We attempted to link each case to a record in the California Birth Registry (CBR; California Department of Public Health, Vital Statistics Branch). A control for each case was randomly selected from the CBR and matched to the corresponding case on date of birth ( \pm 6 months) and sex. Controls were eligible only if they had not been diagnosed with any type of cancer in California prior to the time of diagnosis of the corresponding case.

Calculation of distance from lines. The calculation of distance from lines was blind to the case-control status. Details of the calculation have been described elsewhere (Kheifets et al, 2015). Briefly, mother's residential address at time of birth was obtained from the CBR for both cases and controls and geocoded using an open-source geocoder (Goldberg, 2008). We recorded geocoded latitude and longitude and the geocode-matching geography type, which depended on the completeness of the address. The geocodematching geography types, ordered from smallest to largest median area and, thus, roughly from highest to lowest accuracy in terms of exposure classification, were centroids of tax-assessor parcel, street segment, street, U.S. Postal Service ZIP Code Tabulation Area, town/city, county, and state.

As previously described (Kheifets et al, 2015), a three-tiered approach was used to ascertain straight line distance from home address to nearby overhead transmission lines, with each tier achieving improved distance classification for residences that appeared to be close to lines.

Utility GIS-based distance. We used the geographic information system (GIS) databases of the four largest electric power companies in California to determine the distance from residence to transmission lines over $100 \mathrm{kV}$ within $2000 \mathrm{~m}$. Information on lines with lower voltages (over $60 \mathrm{kV}$ ) was also available for two companies.

Google Earth-based distance. For residences that appeared to have transmission lines within $200 \mathrm{~m}$ according to utility GIS databases or were located in areas not covered by the databases $(7 \%$ of subjects), we measured distances using Google Earth aerial imagery.

Site visit-based distance. For residences of leukaemia cases and controls that appeared to have overhead transmission lines within specified distance criteria based on Google Earth imagery $(<80 \mathrm{~m}$ for $100-200 \mathrm{kV}$ lines, $<150 \mathrm{~m}$ for $200-345 \mathrm{kV}$ lines, and $<200 \mathrm{~m}$ for $500 \mathrm{kV}$ lines), we conducted site visits during which the precise distance of the residence relative to lines was measured. Because of resource constraints, the exposure assessment protocol did not include site visits for CNS cancer cases or controls.

Underground lines were not included in the study.

Variable definitions. Best available distance from birth residence to transmission lines was defined as site visit-based distance, Google Earth-based distance or utility GIS-based distance, in the listed order of precedence as available for each subject. When a residence could not be precisely located within a complex (for example, exact apartment within a building could not be identified), distance from the centre of the complex was used.

Race/ethnicity of parents was obtained from the CBR. We defined child race/ethnicity as White if both parents were White, Black if either parent was Black, Asian if either parent was Asian, Hispanic if either parent was Hispanic and neither parent was Black or Asian, and Other otherwise.

Because variables indicative of socioeconomic status (SES) collected on birth records varied from year to year, we developed a binary SES indicator (high or low) that was based on the father's years of education (high if $>12$ years, low otherwise), mother's years of education (high if $>12$ years, low otherwise), payment method for hospital delivery (low if government programs or no coverage, high otherwise), or a measure of community-based SES derived from U.S. Census data using principal components analysis based on seven indicator variables at the census block level (Yost et al, 2001) (high if $>60$ th percentile of the principal components score, low otherwise), in the listed order of precedence as available 
for each subject. More information on race/ethnicity and SES indicators in CAPS is available in previous publications (Oksuzyan et al, 2012, 2013, 2015a, b).

Statistical analysis. The statistical analysis followed an a priori developed plan. The main analysis considered the shortest distance from the residence to any overhead transmission line $200 \mathrm{kV}$ or higher using best available distance, and was restricted to subjects with geocode-matching geography type of parcel or street segment, which entailed the highest geocoding accuracy. Distances were categorised as in Draper et al (2005), with categories of 0-50, 50$100, \quad 100-200,200-300,300-400,500-600 \mathrm{~m}$, and referent $>600 \mathrm{~m}$. Although cases and controls were matched on sex and age, restriction to parcel or street segment created unmatched subjects; therefore we used unconditional logistic regression controlling for the matching variables to estimate ORs. We further controlled for race/ethnicity (White, Black, Asian, Hispanic, Other) and SES, which were considered important potential confounders. These analyses were conducted for childhood leukaemia (all types) and for CNS cancers (all types). We conducted additional analyses using extended cutpoints that added distance categories of 600 $1000,1000-1500$, and $1500-2000 \mathrm{~m}$ with referent $>2000 \mathrm{~m}$. Further analyses considered the distance to closest line $\geqslant 100 \mathrm{kV}$, distance to closest line of $\geqslant 60 \mathrm{kV}$, and stratification on voltage class of closest line ( $>200,100-200$, and $<100 \mathrm{kV})$.

We conducted subgroup analyses for childhood leukaemia that were: restricted to acute lymphocytic leukaemia cases and controls; stratified by age at diagnosis ( $<5$ years, $\geqslant 5$ years); and stratified by decade of birth (1986-1995, 1996-2006). Sensitivity analyses included: excluding subjects with Down's syndrome, which is associated with higher risk of childhood leukaemia (Mezei et al, 2014); using all subjects regardless of the geocode accuracy; analyses not adjusted for race/ethnicity and SES; conditional logistic regression analyses utilising the case-control matching; and categorising exposure into deciles of distance up to $600 \mathrm{~m}$, with the cutoff points defined by the distribution of distance among the cases. Dose-response trends were tested by the statistical significance of the coefficient for linear trend on the median values of the decile groups among exposed subjects. We also conducted trend analyses based on ranked distance, reciprocal of distance and reciprocal of squared distance, as conducted by Draper et al (2005).

To assess the degree of exposure reclassification associated with the tiered exposure assessment and its impact on risk estimates, we conducted a set of analyses restricted to site-visited residences that compare counts and ORs when using GIS-based distance, Google Earth-based distance, and site visit-based distance.

Analyses were conducted using Stata, version 13 (StataCorp LP, College Station, TX, USA). $P$-values are two-sided.

\section{RESULTS}

Linkage was successful for $87 \%$ of leukaemia cases $(n=5788)$ and $86 \%$ of CNS cancer cases $(n=3308)$. The rate of successful linkage varied by year of diagnosis ( $75 \%$ in $1986,94 \%$ in 2007 ) and by age at diagnosis ( $91 \%$ for ages up to 2 years, $77 \%$ for age 15 years), but not by gender or by race/ethnicity.

Characteristics of study subjects are presented in Table 1. Additional description of the subjects can be found in previous papers (Oksuzyan et al, 2012, 2013). There were 5788 childhood leukaemia and 3308 childhood CNS cancer cases and equal numbers of controls in the study. There were more males than females for both cancers. Race/ethnicity and SES classification were available for most subjects. About $84 \%$ of subjects were geocoded at the street segment or parcel level; thus about $16 \%$ of subjects were excluded from the main analyses due to low-geocoding resolution.

Table 2 presents results of the main analysis, which included subjects with geocode matches of street segment or parcel, used best available distance to nearest transmission line of $200 \mathrm{kV}$ or

Table 1. Characteristics of study subjects, California Power Line Study, 1986-2008

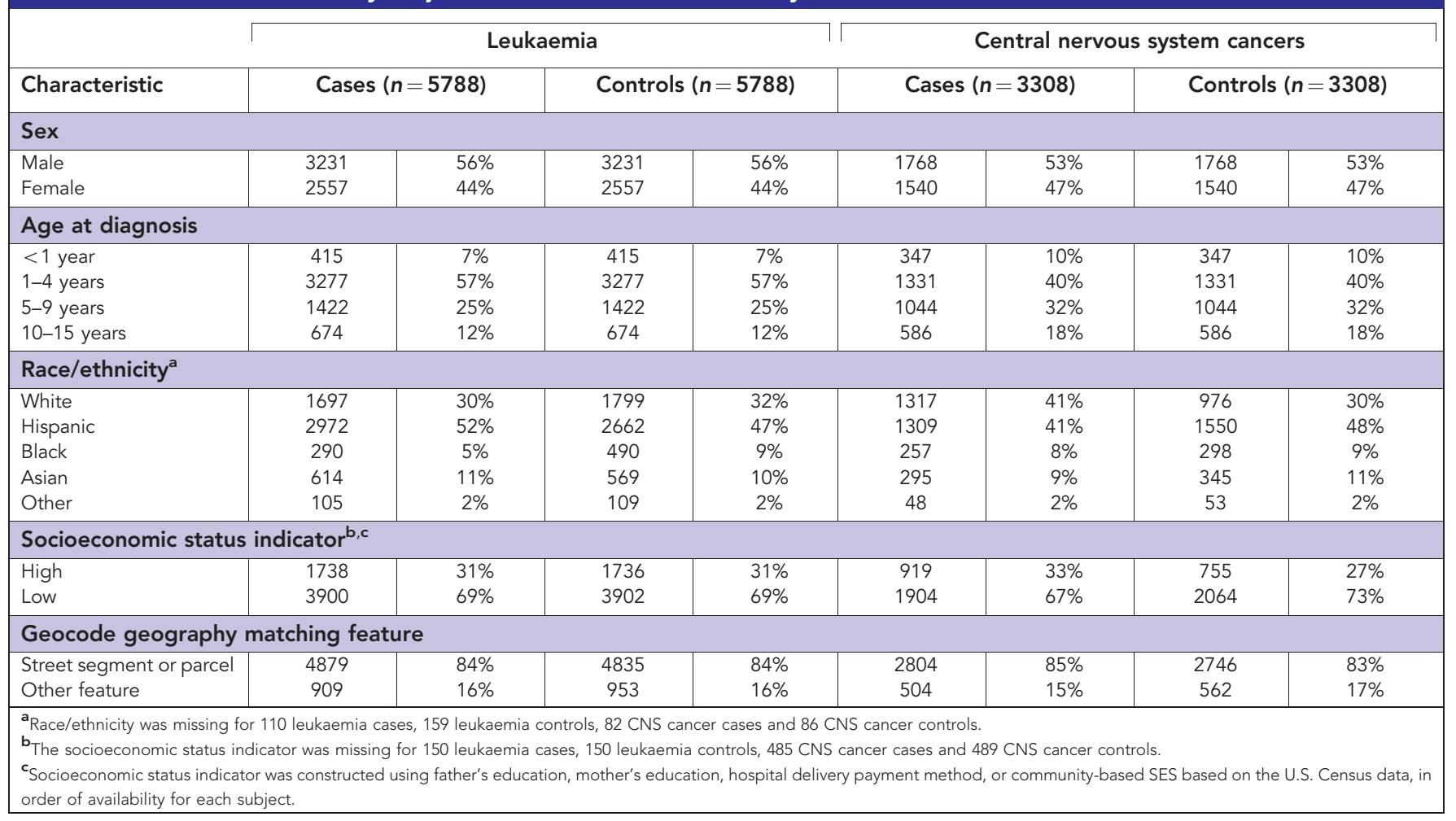


higher, used the same cutpoints and reference group $(>600 \mathrm{~m})$ as Draper et al (2005), and controlled for age, sex, race/ethnicity, and SES. For leukaemia, there was a slight excess of cases at distance of $0-50 \mathrm{~m}$, yielding an adjusted OR of 1.4 (95\% confidence interval: $0.7,2.7)$. ORs for other distances did not show any clear pattern. When using extended distance cutpoints and reference group of $>2000 \mathrm{~m}$ (Figure 1 and Supplementary Table 1), the adjusted OR for distance of $0-50 \mathrm{~m}$ remained 1.4 (95\% confidence interval: 0.7, 2.7), and again, ORs for increasing distance categories showed no pattern. The OR for distance of $600-1000 \mathrm{~m}$ was statistically significantly different from unity $(P=0.01)$. For CNS cancers,

\section{Table 2. Odds ratios for childhood cancer by distance of} residential birth address from closest overhead power transmission line of $200 \mathrm{kV}$ or higher, California Power Line Study, 1986-2008

\begin{tabular}{|c|c|c|c|c|}
\hline Distance, $\mathrm{m}$ & Cases & Controls & OR & $95 \% \mathrm{Cl}$ \\
\hline \multicolumn{5}{|l|}{ Leukaemia } \\
\hline$\geqslant 600$ & 4319 & 4245 & Ref. & \\
\hline $500-600$ & 64 & 53 & 1.2 & $0.8-1.7$ \\
\hline $400-500$ & 52 & 57 & 0.9 & $0.6-1.3$ \\
\hline $300-400$ & 68 & 58 & 1.1 & $0.8-1.6$ \\
\hline 200-300 & 56 & 64 & 0.9 & $0.6-1.2$ \\
\hline $100-200$ & 51 & 66 & 0.8 & $0.5-1.1$ \\
\hline 50-100 & 27 & 27 & 1.0 & $0.6-1.7$ \\
\hline $0-50$ & 22 & 15 & 1.4 & $0.7-2.7$ \\
\hline \multicolumn{5}{|c|}{ Central nervous system cancers } \\
\hline$\geqslant 600$ & 2138 & 2114 & Ref. & \\
\hline $500-600$ & 27 & 25 & 1.1 & $0.6-1.9$ \\
\hline $400-500$ & 40 & 31 & 1.3 & $0.8-2.1$ \\
\hline $300-400$ & 21 & 32 & 0.6 & $0.4-1.1$ \\
\hline 200-300 & 48 & 26 & 1.8 & $1.1-3.0$ \\
\hline 100-200 & 37 & 24 & 1.5 & $0.9-2.6$ \\
\hline 50-100 & 21 & 19 & 1.1 & $0.6-2.0$ \\
\hline $0-50$ & 8 & 7 & 1.2 & $0.4-3.4$ \\
\hline \multicolumn{5}{|c|}{$\begin{array}{l}\text { Abbreviations: } \mathrm{Cl}=\text { confidence interval; } \mathrm{OR}=\text { odds ratio. Analysis restricted to cases and } \\
\text { controls with geocode matches of street segment or parcel. ORs are from logistic } \\
\text { regression models controlling for age, sex, race/ethnicity, and socioeconomic status } \\
\text { indicator. }\end{array}$} \\
\hline
\end{tabular}

there were 7 controls and 8 cases at distance of $0-50 \mathrm{~m}$, yielding an OR of 1.2 (95\% confidence interval: $0.4,3.4$ ) for both referent distances (Table 2; Supplementary Table 1). The OR point estimates exceeded 1 out to a distance of $200-300 \mathrm{~m}$, which had an OR significantly different from unity with both referents (both $P=0.01)$.

When we examined distance to lower-voltage lines, of $100 \mathrm{kV}$ or higher, the ORs for $0-50 \mathrm{~m}$ for both leukaemia and CNS cancer were attenuated to 1.0 , and there was no discernible pattern of association between risk and distance for either cancer type (Table 3). Similar results were obtained when using distance to nearest line of $60 \mathrm{kV}$ or higher (Table 3). ORs for leukaemia stratified by voltage of closest line showed a somewhat elevated OR of 1.4 (95\% confidence interval: 0.7, 2.6) for subjects whose closest line was $>200 \mathrm{kV}$ and who were living within $50 \mathrm{~m}$ of that line, consistent with the main analysis, and no clear association of risk with distance for the lower-voltage strata (Supplementary Figure 1).

Table 4 provides subgroup analyses for childhood leukaemia. When restricting to acute lymphoblastic leukaemia, the OR for 0 $50 \mathrm{~m}$ was 1.3 (95\% confidence interval: $0.6,2.7$ ). In analyses stratified by age at diagnosis, the OR for $0-50 \mathrm{~m}$ was higher for the younger age group (OR of 1.7 for age $<5$ years $v s$ OR of 0.8 for age 5 years and older); the OR for the older age stratum was based on small numbers, however. Analyses stratified by decade of birth yielded a higher $\mathrm{OR}$ for the later decade $(\mathrm{OR}=1.9)$, but with a wide confidence interval (95\% confidence interval: 0.6, 5.4).

Table 5 reports a set of analyses restricted to site-visited residences that compares counts and ORs when using distances based on the three tiers of exposure assessment, which were expected to provide increasingly accurate distance classification. When moving from utility GIS-based to Google Earth-based to site visit-based distance, the number of subjects in the $0-50 \mathrm{~m}$ distance category increased, suggesting improvements in sensitivity. The ORs for those living closest to a high-voltage line also increased with improved exposure classification, but remained imprecise.

Additional sensitivity analyses are reported in Supplementary Table 2. Analysis similar to the main analysis but not adjusting for

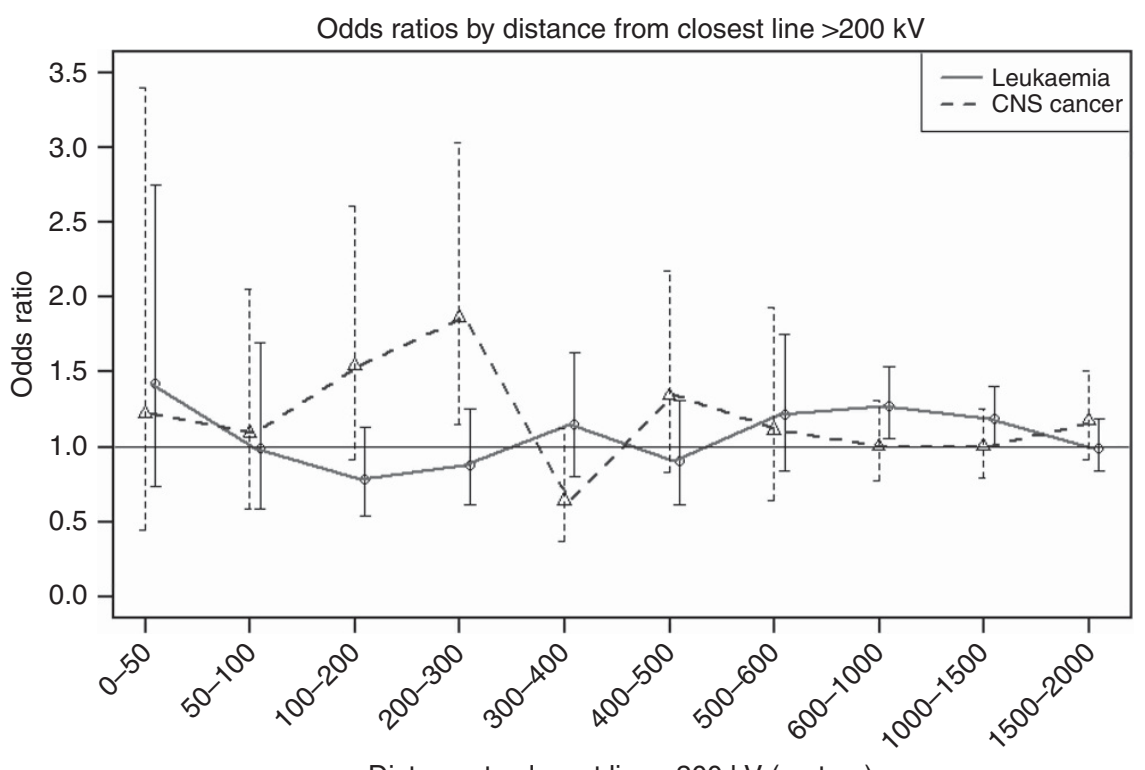

Distance to closest line $>200 \mathrm{kV}$ (metres)

Figure 1. Odds ratios and $95 \%$ confidence intervals for childhood leukaemia and central nervous system cancers by category of distance from the closest transmission line (200 kV or higher). The referent is $2000 \mathrm{~m}$ or more from the lines. The analyses were restricted to cases and controls with geocode matches of street segment or parcel. Odds ratios are from logistic regression models controlling for age, sex, race/ethnicity and socioeconomic status indicator. Distance is based on best available distance. Numeric values are provided in Supplemental Table 1. 
Table 3. Odds ratios for childhood cancer by distance of residential birth address from closest overhead power transmission line of lower voltages, California Power Line Study, 1986-2008

\begin{tabular}{|c|c|c|c|c|c|c|c|c|}
\hline \multicolumn{5}{|c|}{ Distance to closest line $\geqslant 100 \mathrm{kV}$} & \multicolumn{4}{|c|}{ Distance to closest line $\geqslant 60 \mathrm{kV}$} \\
\hline Distance, $\mathrm{m}$ & Cases & Controls & OR & $95 \% \mathrm{Cl}$ & Cases & Controls & OR & $95 \% \mathrm{Cl}$ \\
\hline \multicolumn{9}{|l|}{ Leukaemia } \\
\hline$\geqslant 600$ & 4036 & 3913 & Ref. & & 3776 & 3609 & Ref. & \\
\hline $500-600$ & 99 & 98 & 1.0 & $0.7-1.3$ & 113 & 130 & 0.8 & $0.6-1.1$ \\
\hline $400-500$ & 92 & 105 & 0.9 & $0.6-1.1$ & 122 & 156 & 0.8 & $0.6-1.0$ \\
\hline $300-400$ & 127 & 119 & 1.0 & $0.8-1.3$ & 166 & 169 & 0.9 & $0.8-1.2$ \\
\hline $200-300$ & 102 & 117 & 0.9 & $0.7-1.1$ & 152 & 167 & 0.9 & $0.7-1.1$ \\
\hline $100-200$ & 101 & 138 & 0.7 & $0.5-0.9$ & 156 & 189 & 0.8 & $0.6-1.0$ \\
\hline 50-100 & 54 & 50 & 1.1 & $0.7-1.6$ & 80 & 79 & 1.0 & $0.7-1.3$ \\
\hline $0-50$ & 48 & 45 & 1.0 & $0.7-1.5$ & 94 & 86 & 1.0 & $0.8-1.4$ \\
\hline \multicolumn{9}{|c|}{ Central nervous system cancer } \\
\hline$\geqslant 600$ & 1969 & 1958 & Ref. & & 1820 & 1808 & Ref. & \\
\hline $500-600$ & 55 & 40 & 1.4 & $0.9-2.1$ & 63 & 66 & 1.0 & $0.7-1.4$ \\
\hline $400-500$ & 73 & 47 & 1.6 & $1.1-2.3$ & 95 & 68 & 1.4 & $1.0-2.0$ \\
\hline $300-400$ & 54 & 62 & 0.9 & $0.6-1.3$ & 79 & 86 & 0.9 & $0.7-1.3$ \\
\hline $200-300$ & 70 & 64 & 1.1 & $0.8-1.5$ & 100 & 92 & 1.1 & $0.8-1.5$ \\
\hline $100-200$ & 62 & 55 & 1.1 & $0.8-1.7$ & 95 & 73 & 1.3 & $1.0-1.8$ \\
\hline $50-100$ & 36 & 29 & 1.3 & $0.8-2.1$ & 48 & 39 & 1.2 & $0.8-1.9$ \\
\hline $0-50$ & 21 & 23 & 1.0 & $0.5-1.8$ & 40 & 46 & 0.9 & $0.6-1.4$ \\
\hline
\end{tabular}

race/ethnicity and SES yielded a marginally higher OR of 1.5 for 0-50 m (95\% confidence interval: 0.8, 2.9). Using conditional logistic regression analysis with the original case-control matching yielded an OR of 1.3 (95\% confidence interval: 0.6, 2.7). When including all subjects regardless of geocode accuracy, the OR for $0-50 \mathrm{~m}$ was attenuated to 1.1 (95\% confidence interval: 0.6, 2.1). Excluding subjects with Down's syndrome (36 cases and 4 controls) did not appreciably change results. Post hoc analysis using deciles of distance among exposed cases $<600 \mathrm{~m}$ from lines rather than the pre-specified cutpoints of the main analysis yielded results similar to the main analysis and no evidence of trend $(P=0.40)$. Trend analyses mirroring those in Draper et al (2005) also provided no evidence of trend (all $P$-values $>0.36$ ).

\section{DISCUSSION}

We conducted a large, population-based, record-based, casecontrol study of childhood leukaemia and CNS cancer in California. We found a slightly elevated OR of 1.4 associated with birth residence within $50 \mathrm{~m}$ of transmission lines with voltage $>200 \mathrm{kV}$, which is lower than what was previously reported but still consistent with some prior studies and pooled analysis of distance and magnetic fields. The confidence interval included the null value and there was no evidence of increased risk for farther distances or trend with distance. We did not replicate the finding of Draper et al (2005) of an increased risk of childhood leukaemia extending out to $600 \mathrm{~m}$. Several sporadic statistically significant results were observed at isolated distances $(600-1000 \mathrm{~m}$ for leukaemia, 200-300 $\mathrm{m}$ for CNS cancers). Overall, the pattern of results could not be distinguished from random variation around the null.

In this study, we extended the evaluated distances to power lines out to $2000 \mathrm{~m}$ to evaluate whether the risk extends to farther distances and to explore the impact of a different referent category. Results were virtually identical whether subjects who lived beyond $600 \mathrm{~m}$ or beyond $2000 \mathrm{~m}$ from lines were used as the referent. We also included consideration of lower voltages. There was no association between risk and distance for either leukaemia or CNS cancer for lower-voltage lines.
Our study has a larger number of cases and controls in the highest-exposure category (within $50 \mathrm{~m}$ of $>200 \mathrm{kV}$ lines) compared with other studies of similar design, giving it more statistical power; there were 22 such cases in our study, 9 in the French study (Sermage-Faure et al, 2013), 6 in the updated the United Kingdom study (Bunch et al, 2014), 6 in the Swedish study Kheifets et al (2015), and no cases within $50 \mathrm{~m}$ in the Danish study (Pedersen et al, 2014). We used population-based registries with complete registration of births and cancers, which eliminated participation bias and differential information (recall) bias. Misclassification of outcome status in our study is unlikely due to the completeness and high accuracy of the California Cancer Registry. Controls diagnosed with any type of cancer in California prior to the time of diagnosis of the corresponding case were excluded. Importantly, all aspects of exposure assessment were blind to case/control status.

Other sources of bias such as residual confounding could be present. One potential source of bias is differences in residential mobility between cases and controls. Cases had to be born in and diagnosed in California, whereas controls had to be born in California, but were not required to reside in the state at time of diagnosis of the corresponding case. A study limitation, similar to many studies, is that we have no residential information for controls at the time of diagnosis for index cases. Controls who moved out of California and developed cancer would be misclassified, but mobility combined with a later cancer diagnosis is likely rare. Nevertheless, mobility has been suggested as a potential confounder for the association between childhood leukaemia and magnetic fields exposure from transmission lines (Sahl, 1994). We observed a slightly elevated risk for both leukaemia and CNS cancer for birth addresses within $50 \mathrm{~m}$ of transmission lines; the fact that this occurred for both cancer types when we did not expect any association for CNS cancers may suggest a small bias. However, it is difficult to imagine how differential mobility can induce a higher risk for those living within $50 \mathrm{~m}$ of transmission lines. We plan to conduct a case-only analysis to investigate whether residential mobility could distort risk estimates for the distance to transmission lines or magnetic fields from lines.

About $13 \%$ of cases could not be linked to a California birth record. Because the California Cancer Registry does not collect 
Table 4. Subgroup analyses: odds ratios for childhood leukaemia by distance of residential birth address from closest overhead power transmission line (200 kV or higher), California Power Line Study, 1986-2008

\begin{tabular}{|c|c|c|c|c|}
\hline Distance, $\mathrm{m}$ & Cases & Controls & OR & $95 \% \mathrm{Cl}$ \\
\hline \multicolumn{5}{|c|}{ Acute lymphoblastic leukaemia } \\
\hline$\geqslant 600$ & 3512 & 3475 & Ref. & \\
\hline $500-600$ & 50 & 38 & 1.3 & $0.8-2.0$ \\
\hline $400-500$ & 48 & 50 & 0.9 & $0.6-1.4$ \\
\hline $300-400$ & 59 & 46 & 1.2 & $0.8-1.8$ \\
\hline $200-300$ & 49 & 52 & 0.9 & $0.6-1.4$ \\
\hline $100-200$ & 37 & 50 & 0.7 & $0.5-1.1$ \\
\hline 50-100 & 23 & 21 & 1.1 & $0.6-1.9$ \\
\hline $0-50$ & 16 & 12 & 1.3 & $0.6-2.7$ \\
\hline \multicolumn{5}{|c|}{ Age of diagnosis less than 5 years } \\
\hline$\geqslant 600$ & 2777 & 2737 & Ref. & \\
\hline $500-600$ & 39 & 30 & 1.3 & $0.8-2.1$ \\
\hline $400-500$ & 35 & 37 & 0.9 & $0.6-1.5$ \\
\hline $300-400$ & 41 & 37 & 1.1 & $0.7-1.7$ \\
\hline $200-300$ & 37 & 35 & 1.0 & $0.7-1.7$ \\
\hline 100-200 & 29 & 36 & 0.8 & $0.5-1.3$ \\
\hline 50-100 & 15 & 22 & 0.7 & $0.3-1.3$ \\
\hline $0-50$ & 18 & 10 & 1.7 & $0.8-3.7$ \\
\hline \multicolumn{5}{|c|}{ Age of diagnosis 5 years or older } \\
\hline$\geqslant 600$ & 1542 & 1508 & Ref. & \\
\hline $500-600$ & 25 & 23 & 1.0 & $0.6-1.8$ \\
\hline $400-500$ & 17 & 20 & 0.8 & $0.4-1.6$ \\
\hline $300-400$ & 27 & 21 & 1.2 & $0.7-2.2$ \\
\hline $200-300$ & 19 & 29 & 0.7 & $0.4-1.2$ \\
\hline $100-200$ & 22 & 30 & 0.7 & $0.4-1.2$ \\
\hline 50-100 & 12 & 5 & 2.4 & $0.8-6.9$ \\
\hline $0-50$ & 4 & 5 & 0.8 & $0.2-2.9$ \\
\hline \multicolumn{5}{|c|}{ Birth years $1986-1995$} \\
\hline$\geqslant 600$ & 2544 & 2476 & Ref. & \\
\hline $500-600$ & 41 & 32 & 1.2 & $0.8-2.0$ \\
\hline $400-500$ & 37 & 38 & 0.9 & $0.6-1.5$ \\
\hline $300-400$ & 36 & 38 & 0.9 & $0.6-1.4$ \\
\hline $200-300$ & 33 & 38 & 0.8 & $0.5-1.3$ \\
\hline 100-200 & 30 & 44 & 0.7 & $0.4-1.1$ \\
\hline 50-100 & 15 & 14 & 1.0 & $0.5-2.1$ \\
\hline $0-50$ & 12 & 10 & 1.2 & $0.5-2.7$ \\
\hline \multicolumn{5}{|c|}{ Birth years 1996-2006 } \\
\hline$\geqslant 600$ & 1775 & 1769 & Ref. & \\
\hline $500-600$ & 23 & 21 & 1.1 & $0.6-2.0$ \\
\hline $400-500$ & 15 & 19 & 0.8 & $0.4-1.5$ \\
\hline $300-400$ & 32 & 20 & 1.5 & $0.9-2.7$ \\
\hline $200-300$ & 23 & 26 & 0.9 & $0.5-1.6$ \\
\hline 100-200 & 21 & 22 & 1.0 & $0.5-1.8$ \\
\hline 50-100 & 12 & 13 & 0.9 & $0.4-2.0$ \\
\hline $0-50$ & 10 & 5 & 1.9 & $0.6-5.4$ \\
\hline
\end{tabular}

Abbreviations: $\mathrm{Cl}=$ confidence interval; $\mathrm{OR}=$ odds ratio. Analyses restricted to subjects with geocode matches of street segment or parcel. Odds ratios are from logistic regression models controlling for age, sex, race/ethnicity, and socioeconomic status indicator.

information on US state of birth, we cannot distinguish between cases not linked because they were born outside California from cases not linked because their California birth address was missing or incorrect in the birth records or no longer exists. However, in 1990 and 2010, the percentages of children in California ages of 0 9 years who were born out of state were about 16 and $8 \%$, respectively (Myers, 2013). Because this is roughly in line with our $13 \%$ unsuccessful linkage rate, it is likely that a large portion of the unlinked cases were born outside California. Linkage was less successful for earlier years of diagnosis and older age at diagnosis. This is expected because linkage is more difficult for earlier records, and older cases not only have earlier birth records but were also born in earlier years when a higher proportion of children in California were born outside California.

The accuracy of the utility GIS distance information in terms of distance of residences from transmission lines was generally good
Table 5. Subjects with site visits ${ }^{a}$ : odds ratios for childhood leukaemia by distance of birth address from closest overhead power transmission line $200 \mathrm{kV}$ or higher, California Power Line Study, 1986-2008: Estimates from different exposure assessment tiers

\begin{tabular}{|c|c|c|c|c|}
\hline Distance, m & Cases & Controls & OR & $95 \% \mathrm{Cl}$ \\
\hline \multicolumn{5}{|c|}{ Utility GIS-based distance } \\
\hline$\geqslant 200$ & 46 & 49 & Ref. & \\
\hline $100-200$ & 32 & 36 & 0.9 & $0.4-1.7$ \\
\hline $50-100$ & 21 & 26 & 0.7 & $0.3-1.5$ \\
\hline $0-50$ & 17 & 13 & 1.2 & $0.5-2.9$ \\
\hline \multicolumn{5}{|c|}{ Google Earth-based distance } \\
\hline$\geqslant 200$ & 45 & 49 & Ref. & \\
\hline $100-200$ & 28 & 38 & 0.7 & $0.3-1.4$ \\
\hline $50-100$ & 23 & 23 & 1.0 & $0.5-2.0$ \\
\hline $0-50$ & 20 & 14 & 1.4 & $0.6-3.1$ \\
\hline
\end{tabular}

Site visit-based distance

\begin{tabular}{l|l|l|l|l}
$\geqslant 200$ & 41 & 48 & Ref. & \\
$100-200$ & 28 & 35 & 0.8 & $0.4-1.7$ \\
$50-100$ & 25 & 27 & 1.0 & $0.5-2.0$ \\
$0-50$ & 22 & 14 & 1.6 & $0.7-3.7$
\end{tabular}

Abbreviations: $\mathrm{Cl}=$ confidence interval; $\mathrm{GIS}=$ geographic information system; $\mathrm{OR}=$ odds ratio. Analyses restricted to cases and controls with geocode matches of street segment or parcel. Odds ratios are from logistic regression models controlling for age, sex, race/ ethnicity, and socioeconomic status indicator.

${ }^{a^{a}}$ Site visits were blind to case or control status and did not require participation of subjects.

(Kheifets et al, 2015). However, our three-tiered exposure assessment protocol improved distance classification accuracy, by focusing increasing attention and resources on subjects who appeared to be close to lines, to confirm subject exposure status. The risk increased slightly as the distance accuracy improved. As this is the pattern we would expect to see if a true association existed, this finding could be taken as supportive of a true underlying association. However, the numbers are too small and the increase in risk is too small to consider this a robust finding.

A number of epidemiologic studies have examined the relation between SES and childhood leukaemia. In a review, Poole et al (2006) describe divergent associations between SES and childhood leukaemia, finding negative associations with some individual-level SES indicators such as parental education and positive associations with occupation and community-level SES indicators. Our SES indicator used community-level SES if an individual-level measure was not available. However, none of our SES measures appeared to be associated with increased risk of childhood leukaemia in our study (Oksuzyan et al, 2015).

Most distance studies to date have examined proximity of birth residence to lines, rather than residence at time of diagnosis or other residential history. Reliance on birth residence, or on any other single address, may not necessarily capture all aetiologically relevant exposure. In age-stratified analyses, suggestion of leukaemia risk was limited to the younger age group, for whom birth addresses might be more indicative of lifetime exposure. Among 380 childhood leukaemia cases in a Northern California study, Urayama et al (2009) found that child's residential mobility increased with age, with about $55 \%$ of children $0-4$ years of age having moved at least once compared with $80 \%$ of children $5-14$ years of age. Because acute lymphoblastic leukaemia is a more specific diagnosis and peaks in incidence at ages 2-5 years (Kim et al, 2006), we might expect to find a stronger association for this subtype, should one exist; however, we did not find a stronger association for this diagnosis.

Bunch et al (2014), whose study covered childhood cancer cases diagnosed from 1962 to 2008 in the United Kingdom, found a temporal trend in the risk of childhood leukaemia associated with close proximity to power lines, with the strongest associations present in the earlier decades, 1962-1979. They suggested that the decline might be due to changing population characteristics among 
those living near power lines. We found no evidence for increased risk of leukaemia for earlier time period (birth years 1986-1995) compared with more recent years (1996-2006). However, our study period did not extend as far back in time as the UK study, and we cannot speculate as to the direction or magnitude of risk for earlier years in California. Additional studies may shed light on whether temporal trends are observed in other populations.

A potential limitation of the study is missing data on potential confounders. However, because missing information was largely the result of differences in the information collected on birth certificates from year to year rather than non-response, the potential for biases is small. In fact, our cases and controls were similar on all characteristics considered. We encountered some loss of subjects due to inadequate address information, resulting in poor geocoding accuracy and exclusion from the main analysis. When all subjects regardless of the geocoding accuracy were included, which could be expected to increase exposure misclassification, ORs were close to 1 .

In conclusion, our large, statewide, records-based case-control study of childhood leukaemia and CNS cancer in California provides at most weak support for increased leukaemia risk for children living within $50 \mathrm{~m}$ of transmission lines of above $200 \mathrm{kV}$ reported in other studies and no evidence of risk for those living beyond this distance or near lower-voltage lines.

\section{ACKNOWLEDGEMENTS}

The study was approved by University of California, Los Angeles Office of the Human Research Protection Program, University of Southern California Institutional Review Board, and California Committee for the Protection of Human Subjects. We are extremely grateful to Michael Herz of Pacific Gas and Electric Company, Glenn Sias and Phil Hung of Southern California Edison, Marilyn Dulich of San Diego Gas and Electric Company, Josephine Gonzalez of Los Angeles Department of Water and Power, and other employees of these utilities who contributed generously of their time and provided key data. This work was supported by the Electric Power Research Institute. Crespi was also partially supported by the National Cancer Institute at the National Institutes of Health (grant P30 CA16042).

\section{CONFLICT OF INTEREST}

The authors declare no conflict of interest.

\section{REFERENCES}

Ahlbom A, Day N, Feychting M, Roman E, Skinner J, Dockerty J, Linet M, McBride M, Michaelis J, Olsen JH, Tynes T, Verkasalo PK (2000) A pooled analysis of magnetic fields and childhood leukaemia. Br J Cancer 83(5): 692-698.

Bunch KJ, Keegan TJ, Swanson J, Vincent TJ, Murphy MF (2014) Residential distance at birth from overhead high-voltage powerlines: childhood cancer risk in Britain 1962-2008. Br J Cancer 110(5): 1402-1408.

Draper G, Vincent T, Kroll ME, Swanson J (2005) Childhood cancer in relation to distance from high voltage power lines in England and Wales: a case-control study. BMJ 330(7503): 1290.
Goldberg DW (2008) The USC WebGIS Open Source Geocoding Platform; Los Angeles, CA, USA. Technical Report No. 11. Available at http:// spatial.usc.edu/wp-content/uploads/2014/03/gislabtr111.pdf.

Greenland S, Sheppard AR, Kaune WT, Poole C, Kelsh MA (2000) A pooled analysis of magnetic fields, wire codes, and childhood leukemia. Childhood Leukemia-EMF Study Group. Epidemiology 11(6): 624-634.

Kaune WT, Zaffanella LE (1992) Analysis of magnetic fields far from electric power lines. IEEE Trans Power Del 7: 2082-2091.

Kheifets L, Ahlbom A, Crespi CM, Draper G, Hagihara J, Lowenthal RM, Mezei G, Oksuzyan S, Schüz J, Swanson J, Tittarelli A, Vinceti M, Wunsch Filho V (2010) Pooled analysis of recent studies on magnetic fields and childhood leukaemia. Br J Cancer 103(7): 1128-1135.

Kheifets L, Crespi CM, Hooper C, Oksuzyan S, Cockburn M, Ly T, Mezei G (2015) Epidemiologic study of residential proximity to transmission lines and childhood cancer in California: description of design, epidemiologic methods and study population. J Expo Sci Environ Epidemiol 25(1): 45-52.

Kheifets L, Shimkhada R (2005) Childhood leukemia and EMF: review of the epidemiologic evidence. Bioelectromagnetics 26(S7): S51-S59.

Kim AS, Eastmond DA, Preston RJ (2006) Childhood acute lymphocytic leukemia and perspectives on risk assessment of early-life stage exposures. Mutat Res-Rev Mutat 613(2-3): 138-160.

Mezei G, Sudan M, Izraeli S, Kheifets L (2014) Epidemiology of childhood leukemia in the presence and absence of Down syndrome. Cancer Epidemiol 38(5): 479-489.

Myers D (2013) California's Diminishing Resource: Children. Lucile Packard Foundation for Children's Health. Available at: https://www.usc. edu/schools/price/research/popdynamics/pdf/2013_Myers_CaliforniasDiminishing-Children.pdf.

Oksuzyan S, Crespi CM, Cockburn M, Mezei G, Kheifets L (2012) Birth weight and other perinatal characteristics and childhood leukemia in California. Cancer Epidemiol 36(6): e359-e365.

Oksuzyan S, Crespi CM, Cockburn M, Mezei G, Kheifets L (2013) Birth weight and other perinatal factors and childhood CNS tumors: a case-control study in California. Cancer Epidemiol 37(4): 402-409.

Oksuzyan S, Crespi CM, Cockburn M, Mezei G, Vergara X, Kheifets L (2015a) Race/ethnicity and the risk of childhood leukaemia: a case-control study in California. J Epidemiol Community Health 69(8): 795-802.

Oksuzyan S, Crespi CM, Cockburn M, Mezei G, Vergara X, Kheifets L (2015b) Socioeconomic status and childhood leukemia in California. J Cancer Prev Curr Res 3(4): 00087.

Pedersen C, Raaschou-Nielsen O, Rod NH, Frei P, Poulsen AH, Johansen C, Schuz J (2014) Distance from residence to power line and risk of childhood leukemia: a population-based case-control study in Denmark. Cancer Causes Control 25(2): 171-177.

Poole C, Greenland S, Luetters C, Kelsey JL, Mezei G (2006) Socioeconomic status and childhood leukaemia: a review. Int J Epidemiol 35(2): 370-384.

Sahl JD (1994) Viral contacts confound studies of childhood leukemia and high-voltage transmission lines. Cancer Causes Control 5(3): 279-283.

Sermage-Faure C, Demoury C, Rudant J, Goujon-Bellec S, Guyot-Goubin A, Deschamps F, Hemon D, Clavel J (2013) Childhood leukaemia close to high-voltage power lines-the Geocap study, 2002-2007. Br J Cancer 108(9): 1899-1906.

Urayama KY, Von Behren J, Reynolds P, Hertz A, Does M, Buffler PA (2009) Factors associated with residential mobility in children with leukemia: implications for assigning exposures. Ann Epidemiol 19(11): 834-840.

Wertheimer N, Leeper E (1979) Electrical wiring configurations and childhood cancer. Am J Epidemiol 109(3): 273-284.

Yost K, Perkins C, Cohen R, Morris C, Wright W (2001) Socioeconomic status and breast cancer incidence in California for different race/ethnic groups. Cancer Causes Control 12(8): 703-711.

This work is published under the standard license to publish agreement. After 12 months the work will become freely available and the license terms will switch to a Creative Commons AttributionNonCommercial-Share Alike 4.0 Unported License.

Supplementary Information accompanies this paper on British Journal of Cancer website (http://www.nature.com/bjc) 